\title{
On the extremal function of the modulus of a foliation
}

\author{
MaŁgorzata Ciska-Niedziąomska
}

\begin{abstract}
We investigate the properties of the modulus of a foliation on a Riemannian manifold. We give necessary and sufficient conditions for the existence of the extremal function and state some of its properties. We obtain an integral formula which, in a sense, combines the integral over the manifold with the integral over the leaves. We state a relation between the extremal function and the geometry of the distribution orthogonal to a foliation.
\end{abstract}

Mathematics Subject Classification. 53C12, 58C35.

Keywords. p-modulus, Extremal function, Foliation.

1. Introduction. In 1950 Ahlfors and Beurling [1] introduced a conformal invariant called the extremal length of a family of curves in the plane. Its inverse, called the modulus, plays a major role in the theory of quasiconformal maps. The modulus was generalized to $p$-modulus of families of measures by Fuglede [8] and, in particular, to families of Lipschitz surfaces. In the same paper Fuglede used the notion of $p$-modulus to show completeness of certain classes of functions related to vector analysis. This approach allowed to define the notion of Sobolev spaces on metric measure spaces [12]. On the other hand, the $p$-modulus of families of curves is related to the notion of $p$-capacity of a condenser. The latter is a fruitful tool in potential theory and partial differential equations. It is surprising that the conformal invariance of $p$-capacity for certain choice of $p$ led Ferrand to prove a result on the group of conformal maps on Riemannian manifolds [6].

This article is based on a part of the author's PhD Thesis [11]. The author wishes to thank her advisor Professor Antoni Pierzchalski who suggested the problem under consideration in this paper and for helpful discussions. The author wishes to thank Kamil Niedziałomski for helpful discussions that led to improvements of some of the theorems. In addition, the author wishes to thank anonymous referee for many useful comments, especially the ones included in Remark 3. 
One can show that the $q$-capacity of a condenser (where $q$ is the coefficient conjugate to $p$ ) equals the $q$-modulus of the family of curves joining plates of the condenser and is the reciprocal to the $p$-modulus of the hypersurfaces separating the plates $[9,13]$. Among these surfaces, there is the "smallest" family of surfaces which realizes the modulus. It is given by the level sets of the function realizing the $q$-capacity. Thus this family forms a foliation on the condenser. Moreover, there is a function, called extremal, which realizes this modulus, and the integral of the function on each leaf of the obtained foliation is equal to one [13].

In this short article, we consider a general case of a foliation on a Riemannian manifold. We study properties of the extremal function for the $p$-modulus of a foliation. The majority of results in this paper is obtained under the assumption of existence of the extremal function. We give necessary and sufficient conditions for existence of this function (Theorem 3). In particular, we consider foliations given by the level sets of a submersion (Corollary 6). Moreover, we state some properties of the extremal function.

The existence of the extremal function allows one to define a function

$$
\hat{\varphi}(x)=\int_{L_{x}} \varphi d \mu_{L_{x}}, \quad x \in M,
$$

for any $\varphi \in L^{p}(M)$, where $L_{x}$ is the leaf of $\mathcal{F}$ through $x$. The main result is the following integral formula (Theorem 9)

$$
\int_{M} f_{0}^{p-1} \varphi d \mu_{M}=\int_{M} f_{0}^{p} \hat{\varphi} d \mu_{M},
$$

where $f_{0}$ is the extremal function for the $p$-modulus and $\varphi \in L^{p}(M)$. Moreover, we show that (1) implies that $f_{0}$ is extremal. Using (1), we obtain some results concerning the geometry of a foliation. Namely, the tangent gradient of the extremal function is related to the mean curvature of distribution orthogonal to $\mathcal{F}$.

In the last section we give some examples. We consider a foliation by circles on a torus in $\mathbb{R}^{3}$, a foliation given by the distance function and a foliation by spheres in a ring in $\mathbb{R}^{n}$.

Notice that all results can be generalized to the case of foliations equipped not necessarily with Lebesgue measures but arbitrary Borel measures. Moreover, the "global" Lebesgue measure $\mu_{M}$ could be replaced by any Borel measure. This follows from the original definition of $p$-modulus by Fuglede [8]. In particular, we could consider foliation on a smooth metric measure space as it was pointed out by the anonymous referee. We focus only on the case of Lebesgue measures, since the aim of the author's PhD thesis [11], on which this article is based, was to establish the relation between extrinsic and intrinsic geometry of a foliation and $p$-modulus.

2. Modulus of a foliation. Let $(M, g)$ be a Riemannian manifold, $\mathcal{F}$ a foliation on $M$. Denote by $\mu_{M}$ and $\mu_{L}$ the Lebesgue measures on $M$ and $L \in \mathcal{F}$, 
respectively. Let $L^{p}(M)$ denote the space of all measurable and $p$-integrable functions on $M$ (with the norm $\|\cdot\|_{p}$ ).

Lemma 1. Let $A \subset M$ be a measurable set. Then $\mu_{M}(A)=0$ if and only if

$$
\mu_{M}\left(\left\{x \in M: \mu_{L_{x}}\left(A \cap L_{x}\right)>0\right\}\right)=0 .
$$

In particular, if $f \in L^{p}(M)$ is nonnegative, then the integral $\int_{L} f d \mu_{L}$ exists for almost every leaf $L \in \mathcal{F}$.

Proof. Let $\mathcal{U}=\{U\}$ be a (countable) cover of $M$ such that for each $U \in \mathcal{U}$ there is a submersion $F_{U}: U \rightarrow N_{U}$ with bounded Jacobian such that $F$ defines a foliation $\mathcal{F}$ on $U$. By Fubini's theorem (compare $(2)$ ) there are constants $c_{1}, c_{1}$ (depending on $U$ ) such that

$$
c_{1} \int_{N_{U}} \mu_{L_{y}}\left(L_{y} \cap A \cap U\right) d \mu_{N} \leq \mu_{M}(A \cap U) \leq c_{2} \int_{N_{U}} \mu_{L_{y}}\left(L_{y} \cap A \cap U\right) d \mu_{N},
$$

where $L_{y}=F^{-1}(y)$. Thus $A$ is of $\mu_{M}$-measure zero if and only if the set $\left\{x \in M: \mu_{L_{x}}\left(A \cap L_{x}\right)>0\right\}$ is of $\mu_{M}$-measure zero.

Let $\mathcal{L}$ be an arbitrary subfamily of $\mathcal{F}$. In the space $L^{p}(M)$ consider the family $\operatorname{adm}_{p}(\mathcal{L})$ of all nonnegative functions $f$ such that $\int_{L} f d \mu_{L} \geq 1$ for almost every $L \in \mathcal{L}$. Functions belonging to this family are called admissible. The $p$-modulus of $\mathcal{L}$ is defined as follows

$$
\bmod _{p}(\mathcal{L})=\inf _{f \in \operatorname{adm}_{p}(\mathcal{L})}\|f\|_{p}
$$

if $\operatorname{adm}_{p}(\mathcal{L}) \neq \emptyset$ and $\bmod _{p}(\mathcal{L})=\infty$ otherwise.

We say that $f_{0} \in \operatorname{adm}_{p}(\mathcal{L})$ is extremal for the $p$-modulus of the family $\mathcal{L}$ if $\bmod _{p}(\mathcal{L})=\left\|f_{0}\right\|_{p}$. We will show later (see the considerations preceding Corollary 11) that an extremal function for the $p$-modulus of a foliation (i.e $\mathcal{L}=\mathcal{F}$ ) is unique up to sets of measure zero.

For a family $\mathcal{L} \subset \mathcal{F}$ denote the union of the leaves $L \in \mathcal{L}$ by $\cup \mathcal{L}$. In particular, $\bigcup \mathcal{F}=M$.

Proposition 2 [8]. The modulus has the following properties.

1. If $\mathcal{L} \subset \mathcal{F}$ and $\bigcup \mathcal{L}$ is measurable, then $\bmod _{p}(\mathcal{L}) \leq \bmod _{p}(\mathcal{F})$.

2. If $\mathcal{F}=\mathcal{L}_{1} \cup \mathcal{L}_{2} \cup \cdots$ and $\cup \mathcal{L}_{i}$ is measurable for all $i$, then $\bmod _{p}(\mathcal{F})^{p} \leq$ $\sum_{i} \bmod _{p}\left(\mathcal{L}_{i}\right)^{p}$

3. $\bmod _{p}(\mathcal{L})=0$ if and only if there is $f \in \operatorname{adm}_{p}(\mathcal{L})$ such that $\int_{L} f d \mu_{L}=\infty$ for almost every $L \in \mathcal{L}$.

4. If $f \in L^{p}(M)$, then there is a subfamily $\mathcal{L}$ such that $\bmod _{p}(\mathcal{L})=0$ and $f \in L^{1}(L)$ for almost every $L \in \mathcal{F} \backslash \mathcal{L}$.

5. If $f_{n} \rightarrow f$ in $L^{p}(M)$, then there is a subsequence $\left(f_{n_{i}}\right)$ of $\left(f_{n}\right)$ and a subfamily $\mathcal{L} \subset \mathcal{F}$ such that $\bmod _{p}(\mathcal{L})=0$ and $f_{n_{i}} \rightarrow f$ in $L^{1}(L)$ for almost every $L \in \mathcal{F} \backslash \mathcal{L}$. 
3. Existence and properties of the extremal function. Let $M$ be a Riemannian manifold, $\mathcal{F}$ a foliation on $M$.

Theorem 3. There is an extremal function $f_{0}$ for the $p$-modulus of $\mathcal{F}$ if and only if for any subfamily $\mathcal{L} \subset \mathcal{F}$ such that $\mu_{M}(\bigcup \mathcal{L})>0$, we have $\bmod _{p}(\mathcal{L})>0$.

Proof. $(\Rightarrow)$ Suppose there is a family $\mathcal{L} \subset \mathcal{F}$ such that $\mu_{M}(\bigcup \mathcal{L})>0$ and $\bmod _{p}(\mathcal{L})=0$. By Proposition 2 there is an admissible function $f$ for $\mathcal{L}$ such that $\int_{L} f=\infty$ for almost every leaf $L \in \mathcal{L}$. Moreover, there is some $n>0$ such that $\left\|f_{0}\right\|_{p}>\left\|\frac{1}{n} f\right\|_{p}$ on $\bigcup \mathcal{L}$. Put

$$
\tilde{f}_{0}=\left\{\begin{array}{ll}
f_{0} & \text { on } M \backslash \cup \mathcal{L} \\
\frac{1}{n} f & \text { on } \bigcup \mathcal{L}
\end{array} .\right.
$$

Then $\tilde{f}_{0}$ is admissible for $\mathcal{F}$ but $\left\|f_{0}\right\|_{p}>\left\|\tilde{f}_{0}\right\|_{p}$. Contradiction.

$(\Leftarrow)$ It suffices to show that $\operatorname{adm}_{p}(\mathcal{F})$ is closed in $L^{p}(M)$. Indeed, any closed and convex set in a Banach space contains an element of smallest norm and, as can be easily shown, $\operatorname{adm}_{p}(\mathcal{F})$ is convex. Take a sequence $\left(f_{n}\right)$ of admissible functions converging to $f$. Then $f \in L^{p}(M)$ and $f \geq 0$. By Proposition 2 there is a subsequence $\left(f_{i_{j}}\right)$ and a subfamily $\mathcal{L}$ such that $\bmod _{p}(\mathcal{L})=0$ and $\int_{L} f_{n_{i}} d \mu_{L} \rightarrow \int_{L} f d \mu_{L}$ for every $L \in \mathcal{F} \backslash \mathcal{L}$. Hence $\int_{L} f d \mu_{L} \geq 1$ for every $L \in$ $\mathcal{F} \backslash \mathcal{L}$. Since, by assumption, $\mu_{M}(\bigcup \mathcal{L})=0$, we get that $\int_{L} f d \mu_{L} \geq 1$ for almost every leaf $L \in \mathcal{F}$. Thus $f$ is admissible.

Put

$$
F_{\infty}=\left\{L \in \mathcal{F}: \mu_{L}(L)=\infty\right\} \quad \text { and } \quad M_{\infty}=\bigcup \mathcal{F}_{\infty} .
$$

Corollary 4. If $\mu_{M}(M)<\infty$ and there exists some extremal function for the $p$-modulus of $\mathcal{F}$ for some $p>1$, then $\mu_{M}\left(M_{\infty}\right)=0$.

Proof. Function $f=1$ is admissible for $\mathcal{F}_{\infty}$ and satisfies the condition 3 . of Proposition 2 (for $\left.\mathcal{F}_{\infty}\right)$. Hence $\bmod _{p}\left(\mathcal{F}_{\infty}\right)=0$. Thus, by Theorem 3 , $\mu_{M}\left(M_{\infty}\right)=0$.

We will specify conditions for the existence of an extremal function in the case of a foliation given by the level sets of a submersion.

Let $\Phi: M \rightarrow N$ be a submersion between Riemannian manifolds. Then there is the decomposition

$$
T M=\mathcal{V}^{\Phi} \oplus \mathcal{H}^{\Phi}
$$

where $\mathcal{V}^{\Phi}=\operatorname{ker} \Phi_{*}$ and $\mathcal{H}^{\Phi}=\left(\mathcal{V}^{\Phi}\right)^{\perp}$. The differential $\Phi_{*}: \mathcal{H}^{\Phi} \rightarrow T N$ is a linear isomorphism. Denoting its dual as $\Phi_{*}^{\top}: T N \rightarrow \mathcal{H}^{\Phi}$, we define the Jacobian $J \Phi$ of $\Phi$ as follows

$$
J \Phi=\sqrt{\operatorname{det}\left(\Phi_{*}^{\top} \circ \Phi_{*}: \mathcal{H}^{\Phi} \rightarrow \mathcal{H}^{\Phi}\right)} .
$$

We will need the following version of Fubini's theorem [4]

$$
\int_{M} f d \mu_{M}=\int_{y \in N}\left(\int_{\Phi^{-1}(y)} \frac{f}{J \Phi} d \mu_{\Phi^{-1}(y)}\right) d \mu_{N},
$$


for any nonnegative and measurable function $f$.

Proposition 5. Assume a foliation $\mathcal{F}$ is given by a submersion $\Phi: M \rightarrow N$ such that $J \Phi<C$ for some $C$. Let $\mathcal{L} \subset \mathcal{F}$. If $\bmod _{p}(\mathcal{L})=0$ for some $p>1$, then $\mu_{M}\left(\bigcup\left(\mathcal{L} \backslash \mathcal{F}_{\infty}\right)\right)=0$.

Proof. Suppose $\mu_{M}\left(\bigcup\left(\mathcal{L} \backslash \mathcal{F}_{\infty}\right)\right)>0$. Then there is a subfamily $\mathcal{L}^{\prime}$ of $\mathcal{L}$ such that $\mu_{M}\left(\bigcup \mathcal{L}^{\prime}\right)>0$ and $\mu_{L}(L)<\infty$ for any $L \in \mathcal{L}^{\prime}$. By Proposition 2(3) there is an admisible function $f$ such that $\int_{L} f=\infty$ for almost every leaf $L \in \mathcal{L}$. Thus, by Hölder's inequality, we have

$$
\int_{L} f d \mu_{L}=\int_{L} \frac{f}{(J \Phi)^{\frac{1}{p}}}(J \Phi)^{\frac{1}{p}} d \mu_{L} \leq\left(\int_{L} \frac{f^{p}}{J \Phi} d \mu_{L}\right)^{\frac{1}{p}}\left(\int_{L}(J \Phi)^{\frac{q}{p}} d \mu_{L}\right)^{\frac{1}{q}},
$$

where $\frac{1}{p}+\frac{1}{q}=1$. Hence

$$
\int_{L} \frac{f^{p}}{J \Phi} d \mu_{L}=\infty \text { for almost every } L \in \mathcal{L}^{\prime} .
$$

By (3) and Fubini's theorem (2),

$$
\|f\|_{p}^{p}=\int_{M} f^{p} d \mu_{M}=\int_{N}\left(\int_{\Phi^{-1}(y)} \frac{f^{p}}{J \Phi} d \mu_{\Phi^{-1}(y)}\right) d \mu_{N}=\infty .
$$

Contradiction ends the proof.

Corollary 6. Assume a foliation $\mathcal{F}$ is given by a submersion $\Phi: M \rightarrow N$. Assume $J \Phi<C$ for some $C$ and $\mu_{M}(M)<\infty$. Then there exists some extremal function for the $p$-modulus of $\mathcal{F}$ (for any $p>1$ ) if and only if $\mu\left(M_{\infty}\right)=0$.

Proof. $(\Rightarrow)$ Follows by Corollary 4 .

$(\Leftarrow)$ Assume $\mu\left(M_{\infty}\right)=0$. Take $\mathcal{L} \subset \mathcal{F}$ such that $\bmod _{p}(\mathcal{L})=0$. Then $\mathcal{L} \subset \mathcal{F}_{\infty}$ by Proposition 5 . Hence $\mu(\bigcup \mathcal{L}) \leq \mu\left(M_{\infty}\right)=0$. Therefore Theorem 3 implies that there exists some extremal function for $\mathcal{F}$.

In the end of this section, we state some properties of an extremal function.

Proposition 7. Let $f_{0}$ be some extremal function for the $p$-modulus of a foliation $\mathcal{F}$. Then

1. $\int_{L} f_{0} d \mu_{L}=1$ for almost every leaf $L \in \mathcal{F}$,

2. $f_{0}$ is positive.

Proof. (1) Put $M_{f_{0}}=\left\{x \in M: \int_{L_{x}} f_{0} d \mu_{L_{x}}<\infty\right\}$. By Proposition 2(3) and Theorem 3, $\mu_{M}\left(M \backslash M_{f_{0}}\right)=0$. Hence we may assume $M=M_{f_{0}}$. Consider a function

$$
f(x)=\frac{f_{0}(x)}{\int_{L_{x}} f_{0} d \mu_{L_{x}}}, \quad x \in M .
$$

Then $f$ is admissible, $f \leq f_{0}$, and $\|f\|_{p} \leq\left\|f_{0}\right\|_{p}$. Since $f_{0}$ is extremal, we have $\|f\|_{p}=\left\|f_{0}\right\|_{p}$. Therefore $f=f_{0}$, so $\int_{L} f_{0} d \mu_{L}=1$ for almost every leaf $L \in \mathcal{F}$. 
(2) Suppose there is a set $A$ of positive and finite measure such that $f_{0}=0$ on $A$. Put

$$
\begin{aligned}
& A_{1}=\left\{x \in A: 0<\mu_{L_{x}}\left(L_{x} \cap A\right)<\infty\right\}, \\
& A_{2}=\left\{x \in A: \mu_{L_{x}}\left(L_{x} \cap A\right)=\infty\right\}, \\
& A_{3}=\left\{x \in A: \mu_{L_{x}}\left(L_{x} \cap A\right)=0\right\} .
\end{aligned}
$$

By Lemma $1 \mu_{M}\left(A_{3}\right)=0$. Hence we may assume $A=A_{1} \cup A_{2}$. Let us consider two cases:

(i) $\mu_{M}\left(A_{2}\right)=0$. Then we may assume that $A=A_{1}$. Denote by $B$ the set of all points $x \in M \backslash A$ such that the leaf $L_{x}$ through $x$ meets $A$ on the set of positive measure. For a fixed $0 \leq t \leq 1$ put

$$
f(x)=\left\{\begin{array}{ll}
\frac{1-t}{\mu_{L_{x}}\left(L_{x} \cap A\right)} & x \in A \\
t f_{0}(x) & x \in B \\
f_{0}(x) & x \in M \backslash(A \cup B)
\end{array} .\right.
$$

Then $f \geq 0$ and $\int_{L} f d \mu_{L}=1$ for almost every leaf $L \in \mathcal{F}$. Moreover,

$\|f\|_{p}^{p}=(1-t)^{p} C+t^{p}\left\|\left.f_{0}\right|_{B}\right\|_{p}^{p}+\left\|\left.f_{0}\right|_{M \backslash B}\right\|_{p}^{p}, \quad$ where $C=\int_{A} \frac{1}{\mu_{L_{x}}\left(L_{x} \cap A\right)^{p}} d \mu$.

Hence $\|f\|_{p}<\left\|f_{0}\right\|_{p}$ if and only if the function

$$
\alpha(t)=(1-t)^{p} C+\left(t^{p}-1\right)\left\|\left.f_{0}\right|_{B}\right\|_{p}^{p}, \quad 0 \leq t \leq 1,
$$

is negative at some $t$. Existence of such $t$ follows from the fact that $\alpha(1)=$ 0 and $\alpha^{\prime}(1)=p\left\|\left.f_{0}\right|_{B}\right\|_{p}^{p}>0\left(f_{0}\right.$ does not vanish almost everywhere on $B)$.

(ii) $\mu_{M}\left(A_{2}\right)>0$. Denote by $B$ the set of all points $x \in M \backslash A$ such that the leaf $L_{x}$ through $x$ meets $A_{2}$ on the set of positive measure. For a fixed $0 \leq t \leq 1$ and $\varepsilon>0$, put

$$
f(x)=\left\{\begin{array}{ll}
\varepsilon & x \in A_{2} \\
t f_{0}(x) & x \in B \\
f_{0}(x) & x \in M \backslash\left(A_{2} \cup B\right)
\end{array} .\right.
$$

Then $f \geq 0$ and $\int_{L} f d \mu_{L}=\infty$ if $\mu_{M}\left(A_{2} \cap L\right)>0$ and $\int_{L} f d \mu_{L}=1$ for remaining leaves. Moreover,

$$
\|f\|_{p}^{p}=\varepsilon^{p} \mu_{M}\left(A_{2}\right)+t^{p}\left\|\left.f_{0}\right|_{B}\right\|_{p}^{p}+\left\|\left.f_{0}\right|_{M \backslash B}\right\|_{p}^{p} .
$$

Thus $\|f\|_{p}<\left\|f_{0}\right\|_{p}$ if and only if the function

$$
\alpha(t)=\varepsilon^{p} \mu_{M}\left(A_{2}\right)+\left(t^{p}-1\right)\left\|\left.f_{0}\right|_{B}\right\|_{p}^{p}, \quad 0 \leq t \leq 1,
$$

is negative at some $t$. This is clear by choosing $\varepsilon$ and $t$ small enough (again, as before, $\left\|\left.f_{0}\right|_{B}\right\|_{p}>0$ ).

Remark 1. Proposition 7(1) was first established in [3] but obtained under the assumption of continuity of the extremal function. 
4. The integral formula. Let $M$ be a Riemannian manifold, $\mathcal{F}$ a foliation on $M$. Assume there exists some extremal function $f_{0}$ for the $p$-modulus of $\mathcal{F}$.

Lemma 8. If $\varphi \in L^{p}(M)$, then $\varphi \in L^{1}(L)$ for almost every leaf $L \in \mathcal{F}$.

Proof. It follows immediately by Theorem 3 and Proposition 2(4).

By the above Lemma, for any $\varphi \in L^{p}(M)$ the following function

$$
\hat{\varphi}(x)=\int_{L_{x}} \varphi d \mu_{L_{x}}, \quad x \in M,
$$

is well-defined.

Theorem 9. There is the following integral formula

$$
\int_{M} f_{0}^{p-1} \varphi d \mu_{M}=\int_{M} f_{0}^{p} \hat{\varphi} d \mu_{M}
$$

for any function $\varphi \in L^{p}(M)$.

Proof. Firstly, notice that in the definition of the $p$-modulus we may consider admissible functions which are not necessarily non-negative. Indeed, if $f \in$ $L^{p}(M)$ and $\hat{f} \geq 1$, then its non-negative part $f^{+}$is admissible, since $\hat{f^{+}} \geq$ $\hat{f} \geq 1$. Moreover, $\bmod _{p}(\mathcal{F}) \leq\left\|f^{+}\right\|_{p} \leq\|f\|_{p}$.

Let $\varphi \in L^{p}(M)$. Assume ess $\sup |\hat{\varphi}|<\infty$. Put

$$
\alpha=\varphi-f_{0} \hat{\varphi} \text {. }
$$

Then $\alpha \in L^{p}(M)$ and $\hat{\alpha}=0$. Consider the following one parameter family of functions

$$
f_{t}=f_{0}+t \alpha, \quad t \in \mathbb{R}
$$

Since $f_{0}$ and $\alpha$ are both $p$-integrable, it follows that $f_{t}$ is also $p$-integrable for all $t$. Moreover, by Proposition $7, \hat{f}_{t}=1$. Thus $f_{t}$ is admissible. Fix $\varepsilon \in(0,1)$ and consider a set $M_{\varepsilon}=\left\{x \in M: f_{0}(x)+\varepsilon \alpha(x)>0\right\}$. Fix $x \in M_{\varepsilon}$ and let $\beta(t)=\left|f_{t}(x)\right|^{p}=\left(f_{0}(x)+t \alpha(x)\right)^{p}, t \in(0, \varepsilon)$. By the mean value theorem, there exists $\theta(x) \in(0, t)$ such that $\beta(t)-\beta(0)=t \beta^{\prime}(\theta(x))$, hence

$$
\frac{1}{t}\left(\left|f_{t}(x)\right|^{p}-\left|f_{0}(x)\right|^{p}\right)=p\left(f_{0}(x)+\theta(x) \alpha(x)\right)^{p-1} \alpha(x), \quad x \in M_{\varepsilon} .
$$

Since $M_{\varepsilon} \rightarrow M$ almost everywhere as $\varepsilon \rightarrow 0$, then, by admissibility of $f_{t}$,

$$
0 \leq \int_{M_{\varepsilon}} \frac{1}{t}\left(\left|f_{t}\right|^{p}-\left|f_{0}\right|^{p}\right) d \mu_{M}=p \int_{M_{\varepsilon}}\left(f_{0}+\theta \alpha\right)^{p-1} \alpha d \mu_{M}
$$

for $\varepsilon$ sufficiently small. Notice that the function $\theta$ is measurable, as $f_{t}, f_{0}$, and $\varphi$ are measurable. By the inequality

$$
\left(f_{0}+\theta \alpha\right)^{p-1} \alpha \leq\left(f_{0}+\alpha\right)^{p-1} \alpha \in L^{p}(M)
$$


which follows from the fact that $\theta \in(0,1)$ and the dominated convergence theorem, we get

$$
0 \leq \int_{M} f_{0}^{p-1} \alpha d \mu_{M}
$$

Replacing $\alpha$ by $-\alpha$, we get (4).

Let us now get rid of the assumption ess $\sup |\hat{\varphi}|<\infty$. Let $\varphi \in L^{p}(M)$, and put $M_{n}=\{x \in M:|\hat{\varphi}(x)| \leq n\}$. Define $\varphi_{n}$ equal to $\varphi$ on $M_{n}$ and $f_{0}$ outside $M_{n}$. Then, by the first part of the proof,

$$
\int_{M} f_{0}^{p-1} \varphi_{n} d \mu_{M}=\int_{M} f_{0}^{p} \hat{\varphi_{n}} d \mu_{M}
$$

Hence, by Proposition 7,

$$
\int_{M_{n}} f_{0}^{p-1} \varphi d \mu_{M}=\int_{M_{n}} f_{0}^{p} \hat{\varphi} d \mu_{M}
$$

By the absolute continuity of the Lebesgue integral, we get (4).

Remark 2. We wish to notice that Theorem 9 can be obtained with the use of Badger's general criterion for the existence of the extremal function for the Fuglede $p$-modulus [2], and, in fact, the proofs are similar in a sense. The "original" proof by the author of [11] is more elementary but with a little bit stronger assumptions.

Notice that (4) forces $f_{0}$ to be extremal for the $p$-modulus of $\mathcal{F}$, assuming that an extremal function exists. Indeed, assume (4) holds, where $f_{0}$ is some admissible function for the $p$-modulus of $\mathcal{F}$. Let $\varphi$ be admissible. By (4) and Hölder's inequality, we have

$$
\int_{M} f_{0}^{p} d \mu_{M} \leq \int_{M} f_{0}^{p} \hat{\varphi} d \mu_{M}=\int_{M} f_{0}^{p-1} \varphi d \mu_{M} \leq\left(\int_{M} f_{0}^{p} d \mu_{M}\right)^{\frac{1}{q}}\|\varphi\|_{p} .
$$

Hence

$$
\left\|f_{0}\right\|^{p} \leq\|\varphi\|_{p}
$$

Therefore $f_{0}$ is extremal for the $p$-modulus of $\mathcal{F}$. Summarizing we have the following characterization.

Corollary 10. Assume there is some extremal function for the p-modulus of $\mathcal{F}$. Then the admissible function $f_{0}$ is extremal if and only if the integral formula (4) holds.

Moreover, it is well known that the extremal function is unique (up to sets of measure zero). This follows by Clarkson's inequality. We show how to prove uniqueness of $f_{0}$ with the use of Proposition 7 and Corollary 10. Indeed, assume $f_{0}$ and $g_{0}$ are both extremal for the $p$-modulus of $\mathcal{F}$. Then

$$
\int_{M} f_{0}^{p-1} g_{0} d \mu_{M}=\int_{M} f_{0}^{p} \hat{g_{0}} d \mu_{M}=\int_{M} f_{0}^{p} d \mu_{M}=\left\|f_{0}^{p-1}\right\|_{q}\left\|g_{0}\right\|_{p},
$$


where $\frac{1}{p}+\frac{1}{q}=1$, since $\left\|g_{0}\right\|_{p}=\bmod _{p}(\mathcal{F})$ and $\left\|f_{0}^{p-1}\right\|_{q}=\bmod _{p}(\mathcal{F})^{\frac{p}{q}}$. By Hölder's inequality $g_{0}$ and $f_{0}^{p-1}$ are Hölder dependent. Thus $f_{0}^{p}=f_{0}^{(p-1) q}=$ $c g_{0}^{p}$ for some positive constant $c$. Since $\left\|f_{0}\right\|_{p}=\left\|g_{0}\right\|_{p}$, we get $f_{0}=g_{0}$.

As a next corollary we obtain the formula, firstly proven in [10] using a different approach, for $p$-modulus and the extremal function $f_{0}$ of a foliation given by the level sets of a submersion.

Corollary 11. Assume there is the extremal function $f_{0}$ for the p-modulus of $\mathcal{F}$. If $\mathcal{F}$ is given by a submersion $\Phi: M \rightarrow N$, then

$$
f_{0}=\frac{J \Phi^{\frac{1}{p-1}}}{\widehat{J \Phi^{\frac{1}{p-1}}}} \text { and } \bmod _{p}(\mathcal{F})=\left(\int_{N}\left(\widehat{J \Phi^{\frac{1}{p-1}}}\right)^{1-p} d \mu_{N}\right)^{\frac{1}{p}}
$$

Proof. By Fubini's theorem (2), for any $p$-integrable function $\varphi$, we have ( $q$ is conjugate to $p$, i.e $(p-1)(q-1)=1)$

$$
\begin{aligned}
\int_{M}\left(\frac{J \Phi^{q-1}}{\widehat{J \Phi^{q-1}}}\right)^{p-1} \varphi d \mu & =\int_{M} \frac{J \Phi}{\widehat{J \Phi^{q-1}} p-1} \varphi d \mu_{M} \\
& =\int_{N} \int_{\Phi^{-1}(y)} \frac{\varphi}{{\widehat{J \Phi^{q-1}}}^{p-1}} d \mu_{\Phi^{-1}(y)} d \mu_{N} \\
& =\int_{N} \frac{\widehat{\varphi}}{{\widehat{J \Phi^{q-1}}}^{p-1}} d \mu_{N} .
\end{aligned}
$$

On the other hand,

$$
\begin{aligned}
\int_{M}\left(\frac{J \Phi^{q-1}}{\widehat{J \Phi^{q-1}}}\right)^{p} \widehat{\varphi} d \mu_{M} & =\int_{N} \int_{\Phi^{-1}(y)} \frac{J \Phi^{q-1}}{{\widehat{J \Phi^{q-1}}}^{p}} \widehat{\varphi} d \mu_{\Phi^{-1}(y)} d \mu_{N} \\
& =\int_{N} \frac{\widehat{\varphi}}{{\widehat{J \Phi^{q-1}}}^{p-1}} d \mu_{N} .
\end{aligned}
$$

Therefore formula (4) holds with $f_{0}=\frac{J \Phi^{q-1}}{\widehat{J \Phi^{q-1}}}$. Thus, by Corollary 10, $f_{0}$ is extremal for the $p$-modulus of $\mathcal{F}$.

Remark 3. It seems that we can derive the formula for the extremal function in a more general case. This follows from the fact that the formula (5) does not depend on a choice of the Riemannian metric on $N$.

Assume that the holonomy of $\mathcal{F}$ is finite. The normal bundle to $\mathcal{F}$ can be treated as the quotient $T M / T \mathcal{F}$. Moreover, we have a natural projection $\Phi_{*}$ : $T M \rightarrow T M / T \mathcal{F}$. Since the holonomy is finite, there is a holonomy invariant metric on $T M / T \mathcal{F}$. Thus we may speak about $J \Phi$.

If additionally the leaf space $M / \mathcal{F}$ is Hausdorff, then it has a structure of an orbifold and the natural projection $\Phi: M \rightarrow M / \mathcal{F}$ is a morphism of orbifolds [14]. Up to sets of measure zero, $\Phi$ is smooth, and we may consider the differential $\Phi_{*}$ and, again, define $J \Phi$. 
By the integral formula (4), we obtain some results concerning the geometry of a foliation.

Corollary 12. Let $\mathcal{F}$ be a foliation on $M$ with closed leaves. Assume that the extremal function $f_{0}$ for the p-modulus of $\mathcal{F}$ exists and is $C^{2}$-smooth. Then the mean curvature $H_{\mathcal{F} \perp}$ of the distribution $\mathcal{F}^{\perp}$ orthogonal to $\mathcal{F}$ is of the form

$$
H_{\mathcal{F} \perp}=(p-1)\left(\nabla\left(\ln f_{0}\right)\right)^{\top} .
$$

In particular, $f_{0}$ is constant on leaves of $\mathcal{F}$ (hence $f_{0}=\frac{1}{\hat{1}}$ ) if and only if the distribution $\mathcal{F}^{\perp}$ is minimal.

Proof. Let $X$ be any compactly supported vector field tangent to $\mathcal{F}$. Denote by $\operatorname{div}_{\mathcal{F}} X$ the divergence of $X$ with respect to the leaves of $\mathcal{F}$. Then

$$
\operatorname{div}_{\mathcal{F}} X=\operatorname{div} X+\left\langle H_{\mathcal{F}^{\perp}}, X\right\rangle .
$$

Since for any smooth function $f$ we have

$$
\operatorname{div}(f X)=f \operatorname{div} X+\langle\nabla f, X\rangle,
$$

then putting $\varphi=\operatorname{div}_{\mathcal{F}} X$ in (4), we get

$$
\begin{aligned}
0 & =\int_{M}\left(f_{0}^{p-1}(\operatorname{div} X)+f_{0}^{p-1}\left\langle H_{\mathcal{F}^{\perp}}, X\right\rangle\right) d \mu \\
& =\int_{M}\left(\operatorname{div}\left(f_{0}^{p-1} X\right)-\left\langle\nabla f_{0}^{p-1}, X\right\rangle+f_{0}^{p-1}\left\langle H_{\mathcal{F} \perp}, X\right\rangle\right) d \mu \\
& =\int_{M}\left(f_{0}^{p-1}\left\langle H_{\mathcal{F} \perp}, X\right\rangle-(p-1) f_{0}^{p-2}\left\langle\nabla f_{0}, X\right\rangle\right) d \mu \\
& =\int_{M} f_{0}^{p-2}\left\langle f_{0} H_{\mathcal{F}^{\perp}}-(p-1) \nabla f_{0}, X\right\rangle d \mu .
\end{aligned}
$$

Taking $X=\psi\left(f_{0} H_{\mathcal{F} \perp}-(p-1) \nabla f_{0}\right)^{\top}$, where $\psi$ is a function on $M$ with compact support, we get (6). Moreover, by (6), $\mathcal{F}^{\perp}$ is minimal if and only if $f_{0}$ is constant along leaves, hence $f_{0}=\frac{1}{1}$.

Corollary 13. Let $\mathcal{F}$ be a Riemannian foliation on $M$ with closed leaves. Assume that the extremal function $f_{0}$ for the $p$-modulus of $\mathcal{F}$ exists and is $C^{2}$ smooth. Then $f_{0}$ is constant on leaves of $\mathcal{F}$.

Proof. Follows from the Corollary 12 and the fact that the distribution orthogonal to Riemannian foliation is totally geodesic, hence minimal.

5. Examples. In this section we present some examples, in which we derive formulae for the extremal functions. Moreover, we show how the geometry of a foliation is reflected in the behaviour of the extremal function. This justifies the use of Corollary 13.

The search for the "best" foliation on a Riemannian manifold or the best level sets in the doubly-connected set based on $p$-modulus approach has been considered by the author of [11]. The approach involves considering the variation of $p$-modulus functional and finding foliations being critical points of 
this functional. The results in this article are crucial in those considerations. Here we only demonstrate simple examples of "best" foliations and extremal functions they induce.

5.1. Foliation by circles on a torus in $\mathbb{R}^{3}$. Consider a torus $\mathbb{T}$ in $\mathbb{R}^{3}$ given parametrically

$$
(\alpha, \beta) \mapsto((R+r \cos \alpha) \cos \beta,(R+r \cos \alpha) \sin \beta, r \sin \alpha),
$$

and let $\mathcal{F}$ be a foliation on $\mathbb{T}$ given by the submersion $\Phi(\alpha, \beta)=\beta$. Then $\mathcal{F}$ is a foliation by circles of radius $r$. After some computations we get that the extremal function $f_{0}$ for the $p$-modulus of $\mathcal{F}$ is of the form

$$
f_{0}=C(R+r \cos \alpha)^{\frac{-1}{p-1}}
$$

for some positive constant $C$. Hence, by Corollary 12 ,

$$
H_{\mathcal{F} \perp}=\frac{\sin \alpha}{R+r \cos \alpha} \frac{1}{r} \frac{\partial}{\partial \alpha},
$$

which is the curvature of the orthogonal foliation $\mathcal{F}^{\perp}$ by circles of radii $R+$ $r \cos \alpha$ on the torus $\mathbb{T}$.

5.2. Foliation by a distance function. Let $L_{0}$ be a closed hypersurface in $\mathbb{R}^{n}$. Let $U$ be any of the two connected components of $\mathbb{R}^{n} \backslash L_{0}$. Consider a distance function from $L_{0}$,

$$
\rho(x)=\operatorname{dist}\left(x, L_{x}\right), \quad x \in \mathbb{R}^{n} .
$$

Let $\mathcal{F}$ be a family of level sets of $\rho$. There is a neighborhood $M$ of $L_{0}$ in $U$, in which $\rho$ is smooth and $\mathcal{F}$ is a foliation on $M$, see [7]. Moreover, $|\nabla \rho|=1$ on $M[5]$. By Corollary 11 the extremal function for the $p$-modulus of $\mathcal{F}$ is of the form

$$
f_{0}(x)=\frac{1}{\hat{1}(x)},
$$

hence is constant on the leaves. Clearly, $\mathcal{F}$ is Riemannian and the orthogonal distribution $\mathcal{F}^{\perp}$ is totally geodesic (compare the Corollary 13).

In particular, if $M=\left\{x \in \mathbb{R}^{n}: r_{1}<|x|<r_{2}\right\}$ is a ring and the foliation $\mathcal{F}$ is given by the spheres of radii $r \in\left(r_{1}, r_{2}\right)$, then (in spherical coordinates)

$$
f_{0}\left(r, \alpha_{1}, \ldots, \alpha_{n-1}\right)=C r^{1-n},
$$

for some constant $C$ depending on the dimension $n$.

Open Access. This article is distributed under the terms of the Creative Commons Attribution 4.0 International License (http://creativecommons.org/licenses/ by/4.0/), which permits unrestricted use, distribution, and reproduction in any medium, provided you give appropriate credit to the original author(s) and the source, provide a link to the Creative Commons license, and indicate if changes were made. 


\section{References}

[1] L. Ahlfors and A. Beurling, Conformal invariants and function-theoretic null-sets, Acta Math. 83 (1950), 101-129.

[2] M. BADGER, Beurling's criterion and extremal metrics for Fuglede modulus, Ann. Acad. Sci. Fenn. Math. 38 (2013), 677-689.

[3] D. Blachowska, A modulus and an extremal form of a foliation, Demonstratio Math. 37 (2004), 939-954.

[4] I. Chavel, Riemannian geometry. A modern introduction, Cambridge University Press, Cambridge, 2006.

[5] M. Delfour And J.-P. Zolesio, Shape analysis via oriented distance functions, J. Funct. Anal. 123 (1994), 129-201.

[6] J. FERRAND, The action of conformal transformations on a Riemannian manifold, Math. Ann. 304 (1996), 277-291.

[7] R. Foote, Regularity of the distance function, Proc. Amer. Math. Soc. 92 (1984), 153-155.

[8] B. Fuglede, Extremal length and functional completion, Acta Math. 98 (1957), 171-219.

[9] J. Hesse, A p-extremal length and p-capacity equality, Ark. Mat. 13 (1975), 131-144.

[10] J. Kalina and A. Pierzchalski, A variation of the modulus of submanifold families, Analytic functions, Kozubnik 1979 (Proc. Seventh Conf., Kozubnik, 1979), Lecture Notes in Math., 798, Springer, Berlin, 1980, 250-257.

[11] M. Ciska, Modulus of pairs of orthogonal foliations, PhD Thesis (in Polish), University of Lodz, Poland, 2012.

[12] N. Shanmugalingam, Newtonian spaces: an extension of Sobolev spaces to metric measure spaces, Rev. Mat. Iberoamericana 16 (2000) 243-279.

[13] W. P. Ziemer, Extremal length and conformal capacity, Trans. Amer. Math. Soc. 126 (1967), 460-473.

[14] N. Zhukova, Local and global stability of compact leaves and foliations, Zh. Mat. Fiz. Anal. Geom. 9 (2013), 400-420.

MaŁgorzata Ciska-Niedzia£omska

Department of Mathematics and Computer Science,

University of Łódź,

Banacha 22,

90-238 Lodz,

Poland

e-mail: mciska@math.uni.lodz.pl

Received: 17 November 2015

Revised: 23 March 2016 